\title{
Analysis of ionospheric parameters by the software system "Aurora"
}

\author{
Yury Polozov*, and Nadezhda Fetisova \\ Institute of Cosmophysical Research and Radio Wave Propagation FEB RAS, 684034, Kamchatsky \\ krai, Paratunka, Russian Federation
}

\begin{abstract}
The paper presents methods of modeling and analysis of ionospheric parameters, which realized in the program system of complex analysis of geophysical parameters "Aurora". The methods allow to analyze of characteristic changes in the ionospheric parameters and allocate the anomalous features during periods of ionospheric disturbances. The algorithm parameters are adapted for analyzing the ionospheric data of the Paratunka station (Kamchatka) and based on results of the estimates (station data of Yakutsk, Gakona, etc. were analyzed). Methods can be applied for the mid-latitude region. The system is implemented in the public domain (http://aurorasa.ikir.ru:8580).
\end{abstract}

The research was supported by RSF Grant, project No 14-11-00194.

\section{Introduction}

The realm of the research concerns the problems of the theory of direct experimental data processing. It is associated with the monitoring and the prediction of the state of near-Earth space. At the present time, the databases of various geophysical parameters (National Geophysical Data Center; MAGBAT; SuperMAG) were formed and provided with the means of primary processing and updating. However, the tasks of effective methods creating for the data analysis, the interpretation of the obtained results and their correspondence to model constructions remain largely open. The influence of solar activity on the magnetosphere and the Earth's ionosphere is complex, many aspects of which have not been sufficiently studied so far [1-6]. The most strong and complex ionospheric disturbances (irregularities) are formed during solar flares and geomagnetic storms. They manifest themselves as significant changes of electron concentration in comparison to some characteristic (calm) level and reflect in the ionospheric parameters [1, 2, 7-9]. Methods and computational algorithms developed by the authors for processing of the ionospheric data and the detection of ionospheric irregularities are described in the present paper. Ionospheric data of IKIR FEB RAS chain located in the northeast of Russia were used for the testing of software tools based on the methods and the algorithms. The software is presented in the paper.

*Corresponding author: up_agent@mail.ru 
A large number of studies have been devoted the problems associated with the monitoring of ionospheric conditions and the detection of ionospheric irregularities [1-18]. The International Refence Ionosphere (IRI) model is the international standart of the Earth's ionosphere model [10-13]. It is based on a wide range of ground and space data. Its parameter estimation accuracy depends significantly on the availability of qualitative registered data for a particular region. The accuracy of the IRI model also depends on the level of solar activity, decreasing with a solar activity increase [8, 10-13]. As the IRI model allows to calculate the monthly averages of the ionospheric parameter data for calm conditions, its aplication do not effective for tasks of ionospheric disturbances detection [8, 10]. The recent developments of empirical models, based on neural networks, allow for a significant improvement of the forecast quality in comparison with the IRI model, as they are easy to implement automatically and flexible enough [7-9, 14, 15, 17]. However, these models belong to the "black box" model class. Therefore, for feature spatial description, long training samples with qualitative data are required, which are prone to over fitting and can lead to unexpected results with very noisy data. Also, the operation of systems, based on neural networks, in real time (or close to it) requires the availability of the current information on a complex of geophysical parameters, which is not always feasible [8, 9]. On the basis of the TEC measurements, Afraimovich E.L., Perevalova N.P., and Kosogorov E.A. $[1,7,16]$ developed an approach and a software system for the global detection and the monitoring of ionospheric disturbances of natural and technogenic origin (such as, solar flares and eclipses, geomagnetic disturbances, earthquakes, missile launches, industrial explosions, etc.). TEC data are recorded using the GPS navigation system signals. As noted by the authors themselves, the method error increases significantly during ionospheric disturbances [1]. Also, the results of the ionospheric irregularities detection depend on the parameters setting of GPS/GLONASS ground-receivers net (spatial and temporal resolution, sensitivity). These parameters have influence with a type of detected irregularities (large-scale, medium-scale, small-scale, etc.), that can be registered by a specific network [7]. Therefore, the accuracy and the efficiency of the described methods depend largery on the availability of reliable and qualitative historical ionospheric data. Also, they are determined by the availability of the current geophysical parameters determining the state of the near-Earth space.

The methods described in the paper are based on a complex approach that combines the traditional methods with modern approaches of pattern recognition and artificial intellect, as well as information technologies and systems. The methods are realized in "Aurora" software system for complex analysis of geophysical parameters. The system is presented in open access on the website at http://aurorasa.ikir.ru:8580 (mirror is http://aurorasa2.ikir.ru:8580). Analysis of the ionospheric parameters is performed on the basis of multicomponent model (MCM) developed by the authors [17, 18]. MCM allows us to study the typical duirnal and season vatiations of ionospheric process and to detect anomalies. Ionospheric anomalies can occur during increased solar and geomagnetic activities, as well as during seismic events on Kamchatka. The MCM construction is described in the papers $[17,18]$ in detail. It is based on a joint application of the wavelet transform methods with traditional autoregressive models (ARIMA models). The MCM is theoretically justified [19]. Therefore, its advantage is the ability to obtaine the predicted data with a given confidence probability. Also, ARIMA methods, underlying of the MCM, allow obtaining quite accurate estimates with limited samples. The comparison of the MCM with IRI model, performed in the paper [17], showed that the MCM allowed a more accurate estimate of the predicted data, especially during the solar maximum. This confirms of the efficiency of the suggested approach.

Another approach, implemented in "Aurora" system's ionospheric component, is based on a combination of the wavelet transform and neural networks. Noise component is 
suppressed on the basis of ionospheric data preprocessing by wavelets. This operation allows improving the efficiency of neural networks for the ionospheric irregularities detection [7]. To more detailed study of the ionospheric parameter dynamics in "Aurora" system, the computational solutions based on the continuous wavelet transform (CWT) are used [17]. The CWT application allows the detection of different scale anomalies in the ionosphere and the estimation of their occurence time, duration, and intensity.

\section{Methods for analysis of ionospheric parameters by the software system "Aurora"}

\subsection{Modeling of ionospheric parameter time variations based on MCM}

Modeling of ionospheric parameters in the program system "Aurora" is performed on the basis of following operations:

2.1.1 Using the multiresolution wavelet decomposition (MRA) [17, 20, 21], the $f_{0} F 2$ time series is represented as components:

$$
f(t)=\sum_{k} c_{-3, k} \phi_{-3, k}(t)+\sum_{k} d_{-3, k} \Psi_{-3, k}(t)+e(t)=f_{l}(t)+f_{2}(t)+e(t)
$$

where $\quad c_{-3, k}=\left\langle f, \phi_{-3, k}\right\rangle, \quad \phi_{-3, k}(t)=2^{-3 / 2} \phi\left(2^{-3} t-k\right), \quad \phi \quad$ is a scaling function, $d_{-3, k}=\left\langle f, \Psi_{-3, k}\right\rangle, \Psi_{-3, k}(t)=2^{-3 / 2} \Psi\left(2^{-3} t-k\right), \Psi$ is a wavelet basis, $j=-3$ corresponds to third level of the wavelet decomposition (decomposition level was determined by algorithm [17]), and $e(t)$ is noise.

The wavelet decomposition (Eq. (1)) is carried out by the orthonormal wavelet basis Daubechies of third order (the basis was determined by minimization of the approximation error) [17].

2.1.2. Using the ARIMA methods [19], the modeling of $f_{1}(t)$ and $f_{2}(t)$ components (see Eq. (1)) is performed as:

$$
f_{1}(t)=\sum_{k=\overline{l, N_{3}^{\mu}}} s_{3, k}^{1} \phi_{-3, k}(t), f_{2}(t)=\sum_{k=\overline{l, N_{3}^{\mu}}} s_{3, k}^{2} \Psi_{-3, k}(t)
$$

where $s_{3, k}^{\mu}=\sum_{l=l}^{p_{3}^{\mu}} \gamma_{3, l}^{\mu} \omega_{3, k-l}^{\mu}+e_{3, k}^{\mu}$ is an estimated $\mu$ th component $(\mu=1,2), p_{3}^{\mu}$ and $\gamma_{3, l}^{\mu}$ are the order and parameters of the $\mu$ th component autoregression, $\omega_{3, k}^{\mu}=\nabla \beta_{3, k}^{\mu}, \beta_{3, k}^{1}=c_{-3, k}$, $\beta_{3, k}^{2}=d_{-3, k}, \quad e_{3, k}^{\mu}$ are the residual errors of the $\mu$ th component model, determined as the difference between the actual and modeled values, and $N_{3}^{\mu}$ is the length of the $\mu$ th component.

For the construction and the estimation of the model parameters for the $f_{1}(t)$ and $f_{2}(t)$ components, we used historical hourly data of the foF2 of Paratunka site (Kamchatka, Russia, Institute of Cosmophysical Research and Radio Wave Propagation FEB RAS (IKIR FEB RAS), $52^{\circ} 58^{\prime} \mathrm{N}, 158^{\circ} 15^{\prime} \mathrm{E}$ ) from 1968 to 2013 . During the MCM identification, we considered the dependence of the ionospheric parameters on seasonal variations of solar radiation, solar activity level (SA) and geomagnetic activity. To obtain 
models describing typical variations of the ionospheric parameters, the time intervals for a relatively calm geomagnetic field (sum of the daily K-indices $\sum K<24$ ), without strong seismic events occurring on Kamchatka (without earthquakes of $\sum K \geq 12$, within a 300 $\mathrm{km}$ radius from the station), were used as estimates. The estimation of the model parameters was carried out separately for high and low levels of solar activity and for different seasons. The solar activity was estimated according to the average monthly radio radiation at a wavelength of $\mathrm{f} 10.7$ (for f10.7 $<100$, the activity was considered low, while for f10.7 > 100, it was considered high). Modeling of the foF2 data in the program system "Aurora" is performed for winter (high and low SA) and summer (high and low SA) seasons. The following models are used:

- for winter (high and low SA):

$s_{3, k}^{I}=-0.62 \cdot \omega_{3, k-1}^{I}-0.63 \cdot \omega_{3, k-2}^{I}+0.36 \cdot \omega_{3, k-3}^{I}+e_{3, k}^{I}(t), s_{3, k}^{2}=-0.97 \cdot \omega_{3, k-1}^{2}-0.93 \cdot \omega_{3, k-2}^{2}+e_{3, k}^{2}(t)$

- for summer (high SA) :

$s_{3, k}^{l}=-0.50 \cdot \omega_{3, k-1}^{l}-0.58 \cdot \omega_{3, k-2}^{l}+e_{3, k}^{l}(t) ; s_{3, k}^{2}=-0.88 \cdot \omega_{3, k-1}^{2}-0.80 \cdot \omega_{3, k-2}^{2}+e_{3, k}^{2}(t)$

- for summer (low SA):

$s_{3, k}^{1}=-0.83 \cdot \omega_{3, k-1}^{1}-0.73 \cdot \omega_{3, k-2}^{l}+e_{3, k}^{l}(t), s_{3, k}^{2}=-0.95 \cdot \omega_{3, k-1}^{2}-0.86 \cdot \omega_{3, k-2}^{2}+e_{3, k}^{2}(t)$ where $s_{3, k}^{1}$ is the estimated component $f_{1}(t)$, and $s_{3, k}^{2}$ is the estimated component $f_{2}(t)$.

2.1.3. The models (see Eq. (2)) describe typical variations of the ionospheric parameters, during the anomalous changes, the model errors increase. Thus, in the program system the detection of anomalies is carried out by the estimation of the model errors:

$$
\begin{gathered}
\varepsilon_{\mu}=\sum_{q=1}^{Q_{\mu}}\left|e_{-3, k+q}^{\mu}\right|>T_{\mu}, \\
e_{-3, k+q}^{\mu}=S_{-3, k+q}^{\mu, \text { fact }}-S_{-3, k+q}^{\mu, \text { model }}
\end{gathered}
$$

where $e_{-3, k+q}^{\mu}$ are the residual errors of the $\mu$ th component model for point $k+q$, $\mu=1,2, q$ is time advance of the data, $Q_{\mu}$ is the length of the data time advance based on the $\mu$ th component model, and $T_{\mu}$ is the threshold value of the $\mu$ th component defining the presence of an anomaly. The threshold $T_{\mu}$ was determined for the Paratunka station (Kamchatka, Russia) as:

1. for winter : $T_{1}=1.37 / 1.22$ (high/low SA), $T_{2}=0.97 / 0.73$ (high/low SA).

2. for summer : $T_{1}=1.60 / 1.30$ (high/low SA), $T_{2}=0.88 / 0.80$ (high/low SA).

2.1.4 In the program system the wavelet reconstruction of the component models $f_{1}(t)=\sum_{k=\overline{l, N_{3}^{\mu}}} s_{3, k}^{1} \phi_{-3, k}(t), f_{2}(t)=\sum_{k=\overline{l, N_{3}^{\mu}}} s_{3, k}^{2} \Psi_{-3, k}(t)$ is carried out to the initial resolution $j=0$ :

$$
f_{l}^{O}(t)=\sum_{k} s_{0, k}^{1} \phi_{0, k}(t), \quad f_{2}^{O}(t)=\sum_{k} s_{0, k}^{2} \Psi_{0, k}(t),
$$

where $s_{0, k}^{\mu}=\left\langle f_{\mu}, b_{0, k}^{\mu}\right\rangle, \mu=1,2, b_{3, k}^{1}=\phi_{-3, k}$ is a scaling function, and $b_{3, k}^{2}=\Psi_{-3, k}$ is a wavelet basis.

The MCM errors, obtained after the wavelet reconstruction, are defined as: 


$$
E_{U_{0}}=\frac{1}{U_{0}} \sum_{\mu=1,2} \sum_{k=1}^{U_{0}}\left(e_{0, k+q}^{\mu}(t)\right)^{2}
$$

where $U_{0}$ is the length of the observation time window for the resolution $j=0$ (in the program, an user sets the value of $U_{0}$ on the basis of parameter «Error calculation window»).

Fig. 1 illustrates the results of the foF 2 data analysis for Paratunka station, performed by the MCM. In the analyzed period (30.05.2013 - 04.06.2013) during the magnetic storm of June 1, 2013, anomalous changes occured in the foF2 data as evidenced by the significant increase of MCM errors (the standart deviation (SD) was 2.5 for the $f_{1}(t)$ component and 4.5 for the $f_{2}(t)$ component).
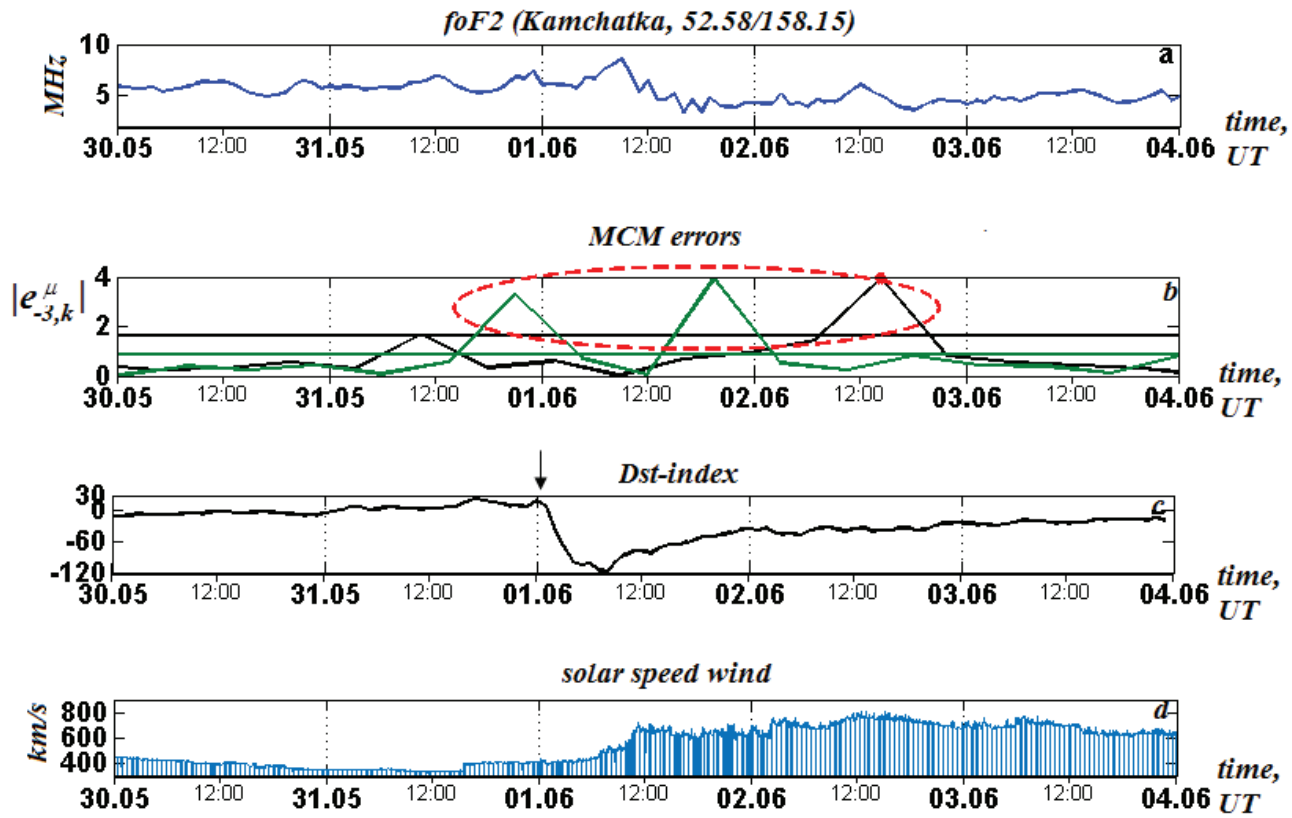

Fig.1. The application of MCM during the magnetic storm on June 1, 2013 (Kamchatka, Russia, time UT): a the foF 2 data, $\mathbf{b}$ MCM errors $\left(f_{1}(t)\right.$ component (black line) and $f_{2}(t)$ component (green line)) and their standard deviations, $\mathbf{c}$ Dst-index of geomagnetic activity, and $\mathbf{d}$ solar wind speed. Red dashed line on graph $b$ indicates the increase of MCM errors during the magnetic storm. On the graph $\mathrm{c}$ the arrow indicates the beginning of the magnetic storm.

\subsection{Approximation of ionospheric data based on the wavelet transform and neural network}

Approximation of the ionospheric parameter time variations in the program system "Aurora" is performed by the following operations:

2.2.1 Using MRA the data time series is represented as $f_{1}(t)$ and $f_{2}(t)$ components (see Eq. (1)).

2.2.2 Using the inverse wavelet transform [21], the reconstruction of the initial resolution $j=0$ is carried out for component $f_{1}(t)=\sum_{k} c_{-3, k} \phi_{-3, k}(t)$ : 


$$
f_{1}^{o}(t)=\sum_{k} c_{0, k} \phi_{0, k}(t)
$$

where $c_{0, k}=\left\langle f_{1}, \phi_{0, k}\right\rangle, \phi_{0, k}(t)=\phi(t-k)$. Modeling of the $f_{1}^{0}(t)$ component is performed on the basis of pre-trained neural networks (NN).

Hourly values of the foF2 from 1968 to 2010 were used for neural networks training. The data with significant gaps were not used in the training, and slight gaps in the data were filled with median values, which calculated for the corresponding hour. To approximation of the foF 2 typical variations, the data for the periods without strong magnetic disturbances and seismic activity in Kamchatka were used during neural networks training. The construction of NN was performed separately for different seasons and different levels of solar activity (see 1.2). The quality criterion of the $\mathrm{NN}$ training was the condition:

$$
E_{A}=\sqrt{e_{A}^{2}(l)}<\varepsilon_{A},
$$

where $\varepsilon_{A}>0$ is predetermined small value, $e_{A}(l)=\hat{c}_{0, l}-c_{0, l}$ is NN error at time $l$, $c_{0, l}$ and $\hat{c}_{0, l}$ are desired and actual output value of the neural network.

The constructed neural networks carry out the advance of the data by the following transformation:

$$
c_{-3, k+1}(t)=\varphi_{3}\left(\sum_{i} \omega_{i j} \varphi_{2}\left(\sum_{q} \omega_{q i} \varphi_{1}\left(\sum_{k} \omega_{k q} c_{-3, k}(t)\right)\right)\right),
$$

where $\omega_{k q}$ are weight numbers of $q$ neuron of the NN outpun layer, $\omega_{q i}$ are weight numbers of $i$ neuron of the NN hidden layer, $\omega_{i j}$ are weight numbers of $j$ neuron of the output layer, $\varphi_{1}(z)=\varphi_{2}(z)=\frac{1}{1+\exp (-z)}$, and $\varphi_{3}(z)=a * z+b$. Configuration of the constructed NN is represented on Fig. 2.

First layer

Second layer

Output layer

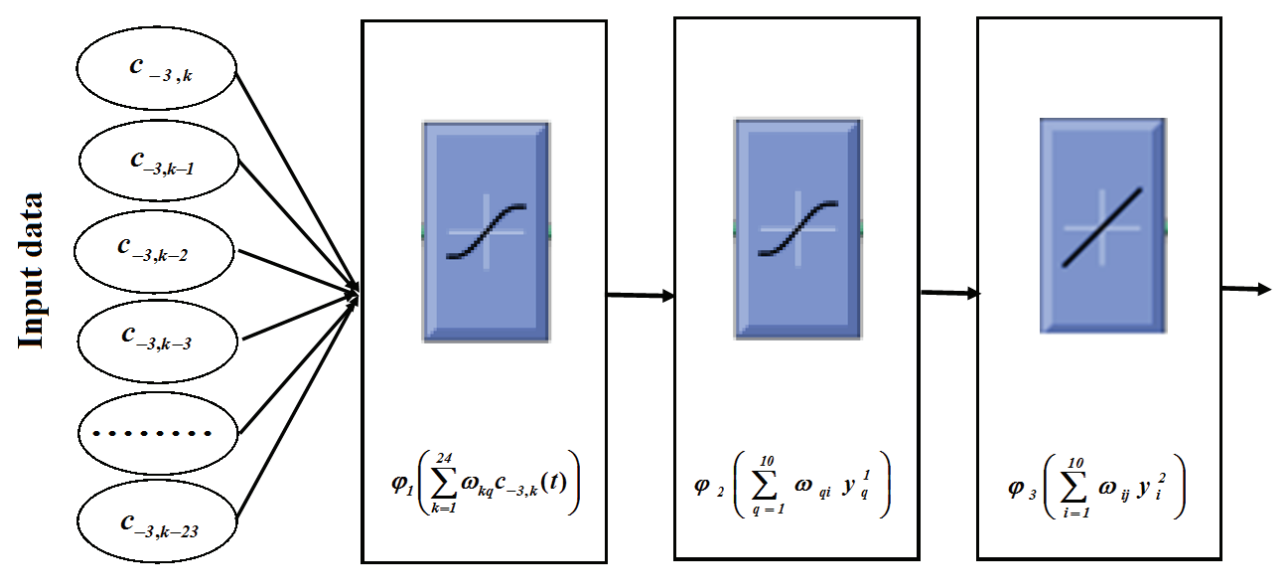

Fig. 2. Configuration of the neural networks 
2.2.3 The trained $\mathrm{NN}$ allows to simulate typical time variations of data. Thus, the procedure of anomalous changes detection may be based on the analyse of the NN errors $e_{A}(l)$.

Fig. 3 illustrates the results of the foF2 data time variation approximation by the NN during the magnetic storm on March 17, 2013. The NN errors do not exceed 0.18 during calm geomagnetic conditions (Fig. 3, graphs c and d). During the magnetic storm, the NN errors significantly increase, indicating the disturbances of the ionospheric parameter typical variations (Fig. 3, graphs c and d).
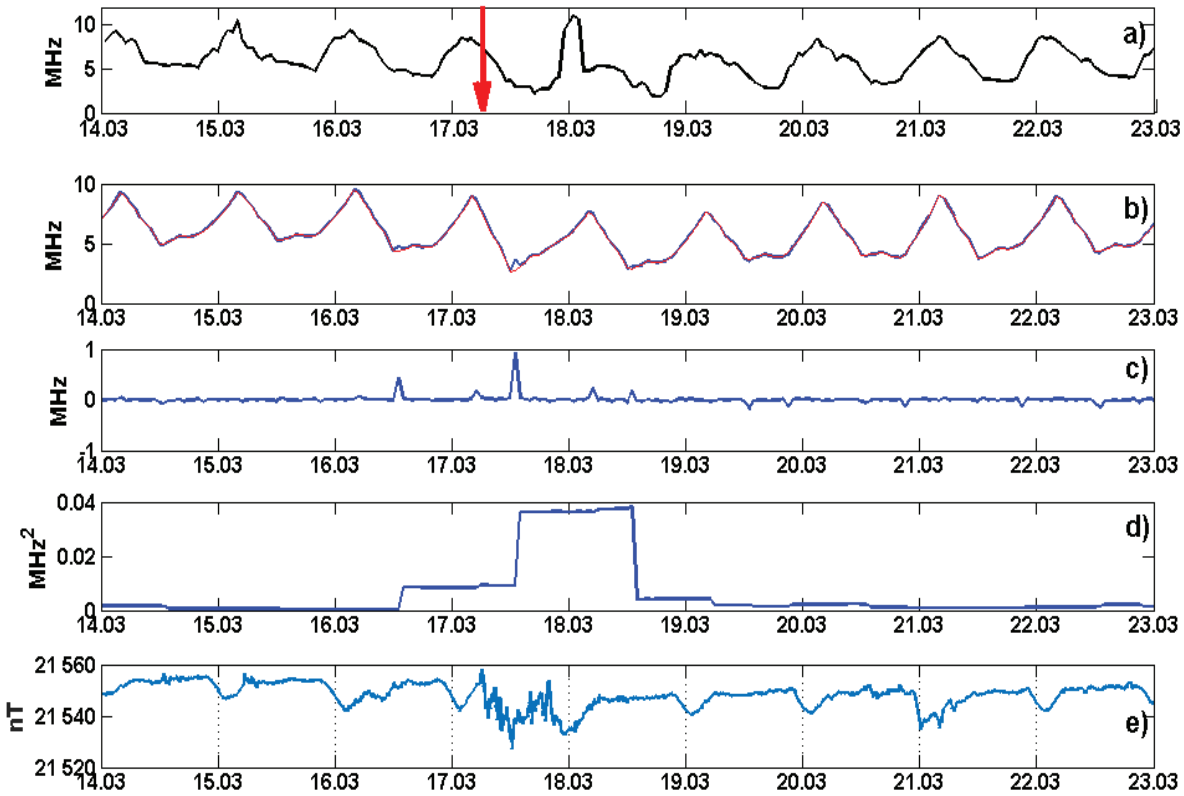

Fig. 3. The results of the foF2 data aproximation by the NN for March 14-22, 2013 : a the foF2 data (Paratunka station), b) the NN output (blue line), the NN aproximation (red line), c) the NN errors, d) - the variance of the NN errors, and e) H-component of the Earth's magnetic field for Paratunka station (it describes the stage of the geomagnetic field). The error variances were estimated in a moving time window with length of 24 samples (it corresponds to the day). The arrow indicates the beginning of the magnetic storm.

\subsection{Anomaly detection in the ionospheric data based on the continuous wavelet transform}

To detailed analysis of the ionospheric parameters in the system "Aurora", we use following computing solution:

1. Performance of the continuous wavelet transform of data:

$$
W_{\Psi} f_{b, a}:=|a|^{-1 / 2} \int_{-\infty}^{\infty} f(t) \Psi\left(\frac{t-b}{a}\right) d t, f \in L^{2}(R), a, b \in R, a \neq 0,
$$

where $a$ is a scale and $\Psi$ is a basis wavelet.

2. Application of a threshold function for the obtained wavelet components $W_{\Psi} f_{b, a}$ : 


$$
P_{T_{a}}\left(W_{\Psi} f_{b, a}\right)=\left\{\begin{array}{l}
W_{\Psi}^{+} f_{b, a}, \text { if }\left(W_{\Psi} f_{b, a}-W_{\Psi} f_{b, a}^{\text {med }}\right) \geq T_{a} \\
0, \text { if }\left|W_{\Psi} f_{b, a}-W_{\Psi} f_{b, a}^{\text {med }}\right|<T_{a} \\
W_{\Psi}^{-} f_{b, a}, \text { if }\left(W_{\Psi} f_{b, a}-W_{\Psi} f_{b, a}^{\text {med }}\right) \leq-T_{a}
\end{array}\right.
$$

where $T_{a}=U * S t_{a}$ is the threshold detects the presence of an anomaly for an $a$ scale (the largest possible scale of decomposition in the program $a_{\max }=150$ ), $S t_{a}=\sqrt{\frac{1}{\Phi-1} \sum_{k=1}^{\Phi}\left(W_{\Psi} f_{b, a}-\overline{W_{\Psi} f_{b, a}}\right)^{2}}$, where $\overline{W_{\Psi} f_{b, a}}$ and $W_{\Psi} f_{b, a}^{\text {med }}$ are the average and median, which, taking into account the diurnal variation of the ionospheric data, are calculated in a moving time window of length $\Phi$ for each hour. $U$ describes a value of the threshold. In the program, an user sets the values of the $\Phi$ and $U$ parameters. The recommended value is $\Phi=168$, which corresponds to a time interval of 7 days. Values of the $U$ parameter for Kamchatka were determined by the estimation of the a posteriori risk, and were

$2.5 \leq U \leq 3.5$ for the data analysis during a high solar activity $\left(\mathrm{f}_{10.7}>100\right)$;

$1.5 \leq U \leq 2.5$ for the data analysis during a low solar activity $\left(\mathrm{f}_{10.7} \leq 100\right)$.

The determined values of $U$ may be recommended for the data analysis of middle latitudes stations.

3. The estimation of the intensity for detected anomalies:

$$
\begin{gathered}
I_{b}^{+}=\sum_{a=1}^{a_{\max }} \frac{\left|P_{T_{a}}^{+}\left(W_{\Psi} f_{b, a}\right)\right|}{\|\left. P_{T_{a}}^{+}\left(W_{\Psi} f_{b, a}\right)\right|_{2}}, \\
I_{b}^{-}=\sum_{a=1}^{a_{\max }} \frac{\left|P_{T_{a}}^{-}\left(W_{\Psi} f_{b, a}\right)\right|}{\|\left. P_{T_{a}}^{-}\left(W_{\Psi} f_{b, a}\right)\right|_{2}},
\end{gathered}
$$

where $\quad\|\|_{2} \quad$ is $\quad$ Euclidian norm, $\quad P_{T_{a}}^{+}\left(W_{\Psi} f_{b, a}\right)=\left|P_{T_{a}}\left(W_{\Psi} f_{b, a}\right)\right|, \quad$ if $\left(W_{\Psi} f_{b, a}-W_{\Psi} f_{b, a}^{m e d}\right) \geq T_{a}, \quad$ and $\quad P_{T_{a}}^{-}\left(W_{\Psi} f_{b, a}\right)=\left|P_{T_{a}}\left(W_{\Psi} f_{b, a}\right)\right|, \quad$ if $\left(W_{\Psi} f_{b, a}-W_{\Psi} f_{b, a}^{\text {med }}\right) \leq-T_{a}$.

Fig. 4 illustrates an example of the aplication for the describing computing solutions. During analyzed period, the strong magnetic storm occured on August 3-4, 2010. On the eve of the magnetic storm, multiscale positive anomaly occured with about from 11.00 UT on August 2 to 10.00 UT on August 4 in the ionospheric data (positive anomaly is shown on Fig. 4 b, in red). The anomaly intensite reached a maximum at 23.00 UT on August 3, 2010 (see Fig. 4 c). In the period from 11.00 to 12.00 UT on August 4, a positive anomaly was replaced by a negative anomaly (a negative anomaly is shown on Fig. 4 b, in blue). Negative anomaly was reached the maximum intensity at 17.00 UT on August 4 and at 10.00 UT on August 5, 2010 (see Fig. 4 c). 

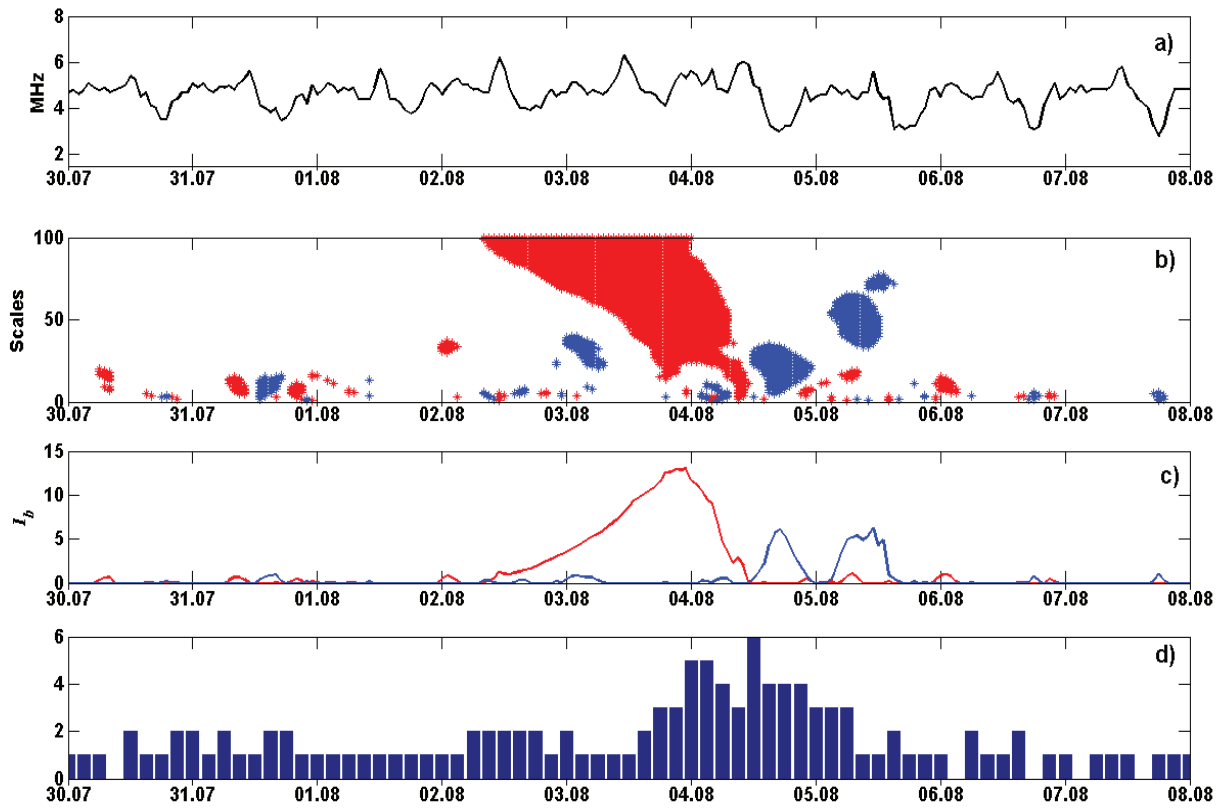

Fig. 4. The results of the foF 2 data processing during the magnetic storm on August 3, $2010:$ a the foF2 data (Paratunka), $\mathbf{b}$ detected anomalies, $\mathbf{c}$ the intensity of anomalies, and $\mathbf{d} \mathrm{K}$-index of the Earth's magnetic field (Paratunka).

\section{Conclusions}

The methods of the ionospheric parameter analisis, presented in the article, are realized in the ionospheric component of the program system "Aurora". The system is available to users on the website at http://aurorasa.ikir.ru:8580. The following functions are realized in the software system:

- Modeling of time variations by the MCM, based on the multiresolution wavelet decompositions and ARIMA methods;

- Approximation of the ionospheric data based on the wavelet transform and neural networks;

- Detection of ionospheric anomalies and the estimation of their intensity.

The algorithm parameters were adapted for the processing of the Paratunka station data $(53.0 \mathrm{~N}, 158.7 \mathrm{E})$. Approbation of the algorithms was also performed and positive results were obtained for Magadan station $(60.0 \mathrm{~N}, 151.0 \mathrm{E})$.

The paper was supported by RSF Grant No. 14-11-00194. The authors are grateful to the organizations carrying out the registration of ionospheric and magnetic data which were applied in the paper.

\section{References}

1. E.L. Afraimovich, N.P. Perevalova, GPS-monitoring verhnej atmosfery Zemli [GPSmonitoring of the Earth's upper atmosphere] (GU NU RVH VSNC SO RAMN, Irkutsk, 2006) 
2. N. I. Budko, A.N. Zaitsev, A.T. Karpachev, A.N. Kozlov, B.P. Filippov, Kosmicheskaya sreda vokrug nas. [The space environment around us] (TROVANT, Troitsk, 2005)

3. O.V. Mandrikova, T.L. Zalyaev, I.S. Solovev, S.Y. Khomutov, D.G. Baishev AOGS13th (2016)

4. O. Mandrikova, I. Solovev, E. Zhizhikina, S. Khomutov, D. Baishev, 26th IUGG, A23p-059 (2015)

5. O.V. Mandrikova, E.A. Zhizhikina, E3S Web of Conferences, 11, (2016)

6. O.V. Mandrikova, I.S. Solovjev, S.Yu. Khomutov, D.G. Baishev, V.V. Geppener, D.M. Klionskiy, Pattern recognition and image analysis, 26, (2016)

7. O. Mandrikova, Yu. Polozov, I. Solovev, N. Fetisova, E3S Web of Conferences, 11, (2016)

8. N.P. Perevalova, Solar-Terrestrial Physics, 19, (2011)

9. M. Nakamura, T. Maruyama, Y. Shidama, Journal of the National Institute of Information and Communications Technology, 56, (2009)

10. K. Watthanasangmechai, P. Supnithi, S. Lerkvaranyu, T. Tsugawa, T. Nagatsuma, T. Maruyama, Earth Planets Space, 64, (2012)

11. D. Bilitza, B.W. Reinisc, Advances in space research, 42, (2007)

12. O.S. Oyekola, P.R. Fagundes, Earth, Planets and Space, 64, (2012)

13. M.V. Klimenko, Earth, Planets and Space, 64, (2012)

14. R.G. Ezquer, J.L. López, L.A. Scidá, M.A. Cabrera, B. Zolesi, C. Bianchi, M. Pezzopane, E. Zuccheretti, M. Mosert, Journal of Atmospheric and Solar-Terrestrial Physics, 107, (2014)

15. X. Zhao, B. Ning, L. Liu, G. Song, Advances in Space Research, 53, (2014)

16. R. Wang, C. Zhou, Z. Deng, B. Ni, Z. Zhao, Journal of Atmospheric and SolarTerrestrial Physics, 92, (2013)

17. E.L. Afraimovich, E.A. Kosogorov, L.A. Leonovich, Earth, Planets, and Space, 52, (2000)

18. O.V. Mandrikova, N.V. Fetisova, Y.A. Polozov, I.S. Solovev, M.S. Kupriyanov, Earth, Planets and Space, 67, (2015)

19. O.V. Mandrikova, N.V. Fetisova (Glushkova), R.T. Al-Kasasbeh, D.M. Klionskiy, V.V. Geppener, M.Y. Ilyash, Annals of Geophysics, 58, 2015

20. G. Box, G. Jenkins, Time series analysis: Forecasting and control (Holden-Day, San Francisco, 1970)

21. S. Mallat, A wavelet tour of signal processing (Academic Press, London, 1999)

22. C.K. Chui, An introduction in wavelets (Academic Press, New York, 1992)

23. A.D. Ageev, Neiromatematika: uchebnoe posobie dlya vyzov [Neuromathematics: Textbook for high schools] (IPRZhR, Moscow, 2002) 